\title{
PRODUTIVIDADE DE GENÓTIPOS DE MARACUJAZEIRO AZEDO SOB DOSES DE POTÁSSIO, NO DISTRITO FEDERAL ${ }^{1}$
}

\author{
AURÉLIO TINOCO DE OLIVEIRA², JOSÉ RICARDO PEIXOTO³, NILTON TADEU VILELA JUNQUEIRA4, \\ LUIZ EDUARDO PACIFICI RANGEL ${ }^{5}$, JULIANA MEIRELES FORTALEZA ${ }^{6}$
}

\begin{abstract}
RESUMO - Objetivando avaliar o efeito de três diferentes níveis de adubação potássica sobre a produção do maracujazeiro azedo (Passiflora edulis Sims e P. edulis Sims f. flavicarpa Deg.), foi realizado um experimento na Fazenda Água Limpa da Universidade de Brasília de dezembro/1999 a maio/2001, utilizando-se o delineamento em blocos casualizados, em esquema fatorial 9x3, sendo nove genótipos e três níveis de adubação potássica, totalizando 27 tratamentos, quatro repetições e nove plantas úteis/parcela. Os genótipos avaliados foram: Híbrido EC-2-0; Marília Seleção Cerrado; $\mathrm{F}_{1}$ (Roxo Fiji x Marília); Porto Rico; Vermelhão ( $\left.\mathrm{RC}_{1}\right) ; \mathrm{F}_{1}$ (Marília x Roxo australiano); Redondão; IAC-273 e Itaquiraí. Os três níveis de adubação potássica aplicados foram de 0,640 e $1280 \mathrm{~kg} \mathrm{de} \mathrm{K}_{2} \mathrm{O} \mathrm{ha}^{-1}$, utilizando-se como fonte o cloreto de potássio. Ao final de cinco meses de colheita os genótipos híbrido EC-2-0 e Marília Seleção Cerrado foram os que apresentaram as maiores produções $\left(21.675\right.$ e $\left.21.577 \mathrm{~kg} \mathrm{ha}^{-1}\right)$ e maiores números de frutos por planta $\left(155,28\right.$ e 149,24), respectivamente. O peso médio de fruto variou de $88 \mathrm{~g}$ (Itaquiraí) até 103,42g ( $\mathrm{F}_{1}$ - Marília x Roxo Australiano). As doses de adubação potássica influenciaram as seguintes variáveis estudadas: produção de frutos de primeira ha' ${ }^{-1}$; produção frutos totais $\mathrm{ha}^{-1}$; peso médio de frutos de primeira e peso médio de frutos $1 \mathrm{~A}$. A interação genótipos $\mathrm{x}$ adubação potássica apresentou diferença estatística para o número de frutos de primeira/planta para uma dosagem de $640 \mathrm{~kg} \mathrm{~K}_{2} \mathrm{O}_{\text {ha }}{ }^{-1}$.
\end{abstract}

Termos de Indexação: Passiflora edulis, P. edulisf.flavicarpa, fertilização, cerrado.

\section{PRODUCTIVITY OF GENOTYPES OF PASSION-FRUIT RELATED TO LEVELS OF POTASSIUM FERTILIZATION IN THE REGION OF BRASÍLIA-BRAZIL}

\begin{abstract}
An experiment was carried out to verify the effect of three levels of a potassium fertilizer on the yield of nine genotypes of passion fruit (Passiflora edulis Sims and P. edulis Sims. f. flavicarpa, Deg.). The experiment was installed at Fazenda Água Limpa, the experiment station of the Universidade de Brasília, from December 1999 to May, 2001. The design was randomized blocks, 27 treatments in a factorial scheme 9x3: nine genotypes of passion fruit and three levels of the potassic fertilizer with four replications and nine plants per plot. The genotypes tested were: Híbrido EC-2-0, Marília Seleção Cerrado, $\mathrm{F}_{1}$ (Roxo Fiji x Marília), Porto Rico, Vermelhão ( $\mathrm{RC}_{1}$ ), $\mathrm{F}_{1}$ (Marília x Roxo Australiano), Redondão, IAC-273 and Itaquiraí. The potassic fertilizer levels were $0 ; 640$ and $1,280 \mathrm{~kg} \mathrm{~K}_{2} \mathrm{O} \mathrm{ha}^{-1}$. The source of potassium was the potassium chloride. The parameters checked were: fruit production $\left(\mathrm{kg} \mathrm{ha}^{-1}\right)$, number of fruits per plant and average fruit weight. After five months of regular harvesting the genotypes Híbrido EC-2-0 and Marília Seleção Cerrado showed the highest production (21,675 and 21,577 kg ha $\left.{ }^{-1}\right)$ and the highest number of fruits per plant $(155,28$ and 149,24$)$ respectively. The average weight of fruits varied from $88 \mathrm{~g}$ (Itaquiraí) to 103,42g F (Marília x Roxo Australiano). The potassium fertilizer levels influenced the following parameters: production of marketable fruits/ha (type 1), average weight of marketable fruits (type 1) and average weight of more marketable fruits (type 1A). The interaction of genotypes versus potassium fertilization showed statistical significance to the number of marketable fruits (type 1) per plant, concerning the second level, $640 \mathrm{~kg} \mathrm{ha}^{-1}$, of potassium chloride.
\end{abstract}

Index terms: Passiflora edulis, P. edulis f. flavicarpa, potassium fertilization.

\section{INTRODUÇ̃̃̃O}

O gênero Passiflora é originário da América do Sul e tem no Centro-Norte do Brasil o maior centro de distribuição geográfica. No Brasil a espécie mais importante é $P$. edulis f. flavicarpa, maracujá amarelo ou azedo. Em pequena escala é cultivado o maracujá doce, $P$. alata Dryand (Manica, 1981). Junqueira et al. (1999) citam, dentre outros fatores, como responsáveis pela baixa produtividade alcançadas na cultura do maracujazeiro no Brasil, o cultivo de genótipos inadequados e a ausência de um plano adequado de adubação. A planta do maracujazeiro apresenta crescimento rápido e contínuo, extraindo assim grande quantidade de nutrientes do solo. O nitrogênio é o nutriente mais absorvido, $205 \mathrm{~kg}_{\text {de }} \mathrm{N} \mathrm{ha}^{-1}$ ano $^{-1}$, seguido pelo potássio, $184 \mathrm{~kg}$ de $\mathrm{K} \mathrm{ha}^{-1} \mathrm{ano}^{-1}$ (Haag et al. 1973). A deficiência de potássio no maracujazeiro ocasiona clorose seguida de necrose a partir das folhas mais velhas, secamento das gavinhas, redução na ramificação, redução no diâmetro dos ramos, queda acentuada de flores e alteração do teor de sólidos solúveis dos frutos (Ruggieroet al., 1996; Quaggio \& Piza Júnior, 1998; Junqueira et al., 1999). O presente trabalho teve como objetivo verificar o desempenho agronômico de nove genótipos de maracujazeiro azedo, cultivados sob três doses de adubação potássica, no Distrito Federal.

\section{MATERIALE MÉTODOS}

O experimento realizou-se na Fazenda Água Limpa (FAL/UnB), situada no Distrito Federal a uma latitude de $16^{\circ}$ Sul, longitude de $48^{\circ}$ Oeste numa altitude de $1.100 \mathrm{~m}$. Aárea total utilizada foi de 0,8 hectare. A precipitação no período compreendido entre abril de 2000 a maio de 2001 , data da última colheita do experimento, foi de $1.818,3 \mathrm{~mm}$. A média da temperatura mínima mensal para o mesmo período foi de $19,32^{\circ} \mathrm{C}$, alcançando a temperatura mínima absoluta de $16,6^{\circ} \mathrm{C}$ nos meses de junho e julho de 2000.

Utilizou-se o delineamento em blocos casualizados, em esquema fatorial $9 \times 3$, sendo nove genótipos e três doses de adubação potássica, totalizando 27 tratamentos, quatro repetições e nove plantas úteis/parcela. Utilizou-se os seguintes genótipos: híbrido EC-2-0, Marília Seleção Cerrado (MSC), $\mathrm{F}_{1}$ (Roxo Fiji x Marília), Porto Rico, Vermelhão, $\mathrm{F}_{1}$ (Marília x Roxo Australiano), Redondão, IAC - 273 e Itaquiraí. As plantas dos noves genótipos foram semeadas no dia 23/09/1999. As mudas foram transplantadas noventa dias após a semeadura. O experimento foi instalado num solo Latossolo Vermelho-Amarelo, fase argilosa, profundo, com boa drenagem e de baixa fertilidade natural, apresentando os seguintes teores de nutrientes: $\mathrm{Al}\left(0,05 \mathrm{cmol}_{\mathrm{c}} \mathrm{dm}^{-3}\right) ; \mathrm{Ca}+\mathrm{Mg}\left(1,9 \mathrm{cmol}_{\mathrm{c}}\right.$

\footnotetext{
${ }^{1}$ (Trabalho 173/ 2002). Recebido: 18/07/2002. Aceito para publicação: 31/10/2002 . Parte da dissertação de Mestrado do primeiro autor.

${ }^{2}$ Eng. Agr. Msc., QNA 09 Cs.19, Taguatinga/DF, 72110-090, Email:aureliotinoco@bol.com.br.

${ }^{3}$ Prof. Dr. da Faculdade de Agronomia e Medicina Veterinária/UnB, Cx. Postal:04508, 70910-900, E-mail:peixoto@unb.br.

${ }_{4}^{4}$ Pesquisador da Embrapa-Cerrados - CPAC, BR-020 Km 18, Planaltina/DF, 73301-970, E-mail:junqueir@cpac.embrapa.br.

${ }^{5}$ Eng. Agr. Msc., Cx. Postal:04508, 70910-900.

${ }^{6}$ Eng. Agr. Msc., SQN 304, Bloco F, apto. 209, Brasília/DF, 70736-060.
} 
$\left.\mathrm{dm}^{-3}\right) ; \mathrm{P}\left(4,5 \mathrm{mg} \mathrm{dm}^{-3}\right) ; \mathrm{K}$ (46 $\left.\mathrm{mg} \mathrm{dm}^{-3}\right) ; \mathrm{pH}$ (água) 5,4 e saturação por Al $4 \%$. Para o Al foi utilizado o extrator $\mathrm{KCl}$ e a titulação com $\mathrm{NaOH}$; para o $\mathrm{Ca}+\mathrm{Mg}$ foi utilizado o extrator $\mathrm{KCl}$ e a titulação com EDTA; para o P e K foi utilizado o extrator Mehlich I (duplo ácido) (Osaki, 1991). Foi realizada uma calagem com calcário dolomítico (2,5 t/ha) em todo o terreno, elevando a saturação por bases inicial de $V=30 \%$ para $V=60 \%$ (Souzaet al. 1999). Foi aplicado $1 \mathrm{~kg}$ de superfosfato simples (Raij et al. 1996) por cova mais carbofuran (386 g do p.a./ha). O espaçamento utilizado foi de $2,75 \times 2,50 \mathrm{~m}$, totalizando 1.454 plantas ha-1 (incluindo a bordadura).

As adubações de cobertura foram realizadas mensalmente, no período compreendido entre janeiro a maio/2000 e setembro/2000 a maio/

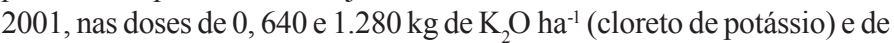
$428 \mathrm{~kg}$ de $\mathrm{N} \mathrm{ha}^{-1}$ (sulfato de amônio). Realizou-se ainda uma única cobertura com 43,6 kg de $\mathrm{P}_{2} \mathrm{O}_{5}$ ha $^{-1}$ (superfosfato simples) em dezembro de 2000 .

O plantio foi conduzido, utilizando-se o sistema de sustentação de espaldeira vertical, sem podas de renovação. Foram realizadas pulverizações com fungicidas sistêmico e preventivo, e com inseticida, no intervalo de 20 dias, a partir do plantio até o início da colheita, para controle de pragas e doenças, utilizando-se os seguintes produtos: óxido cuproso; mancozeb; tebuconazole; benomyl; enxofre; deltamethrin e carbofuran (plantio), nas dosagens recomendadas para a cultura. Não se realizou a polinização artificial.

As colheitas foram realizadas uma vez por semana, recolhendo somente aqueles frutos que se encontravam no chão. Os frutos colhidos foram levados para um armazém onde foram contados e classificados pelo tamanho em relação à medida de seu diâmetro equatorial (DE), com o uso de um paquímetro, e depois pesados. As variáveis analisadas foram: produtividade em $\mathrm{kg} \mathrm{ha}^{-1}$, número de frutos por planta e peso médio dos frutos, de primeira ( $\mathrm{DE}<65 \mathrm{~mm})$, frutos $1 \mathrm{~A}(65<\mathrm{DE}<75 \mathrm{~mm})$, frutos $2 \mathrm{~A}$ $(75<\mathrm{DE}<85 \mathrm{~mm})$, frutos $3 \mathrm{~A}(\mathrm{DE}>85 \mathrm{~mm})$ e frutos totais (Meletti, 1999). A primeira colheita foi realizada no dia 14/12/2000, sendo a última no dia $02 /$ 05/2001, totalizando vinte colheitas. Para análise de produção em $\mathrm{kg} \mathrm{ha}^{-1}$, número de frutos e peso médio dos frutos totais utilizou-se os dados das 20 colheitas. Para avaliação de produção em $\mathrm{kg} \mathrm{ha}^{-1}$, do número de frutos e do peso médio dos frutos de primeira, 1A, 2A, 3A, utilizou-se as onze últimas colheitas. Considerou-se como frutos totais a soma das quatro classificações.

As análises de variância para cada parâmetro, bem como a comparação entre os genótipos pelo teste de Tukey a 5\% de significância, foram executadas com a utilização do "software SANEST", de autoria de Zonta \& Machado (1995). Foram realizadas análises de regressão e correlação entre todas as variáveis avaliadas, baseando-se na significância de seus coeficientes. A classificação de intensidade da correlação para $\mathrm{p}$ $\leq 0,01$ considerou muito forte $(\boldsymbol{r} \pm 0,91$ a $\pm 1,00)$, forte $(\boldsymbol{r} \pm 0,71$ a $\pm 0,90)$, média $(\boldsymbol{r} \pm 0,51$ a $\pm 0,70)$ e fraca $(\boldsymbol{r} \pm 0,31$ a $\pm 0,50)$, de acordo com Gonçalves
\& Gonçalves (1985), citados por Guerra \& Livera (1999).

\section{RESULTADOS E DISCUSSÃO}

Houve diferenças significativas entre os genótipos e na interação genótipo $\mathrm{x}$ doses de potássio, para o número de frutos de primeira (Tabela 1).

Os genótipos EC-2-0 e Porto Rico apresentaram maior número de frutos de primeira por planta, diferindo apenas do Itaquiraí, no

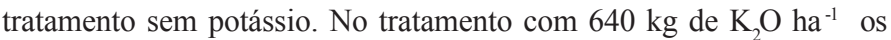
genótipos Vermelhão e Redondão diferiram entre si (Tabela 1). Para o número de frutos 1A, 2A, 3A e frutos totais, com exceção do genótipo Itaquiraí, que apresentou o menor número de frutos, os genótipos não apresentaram diferenças significativas entre si (Tabela 2).

Os genótipos Marília Seleção Cerrado, Porto Rico, Vermelhão, híbrido EC-2-0, $\mathrm{F}_{1}$ (Roxo Fiji x Marília) e $\mathrm{F}_{1}$ (Marília x Roxo Australiano) foram os que apresentaram maior produção de frutos de primeira. Para a produção de frutos $1 \mathrm{~A}, 2 \mathrm{~A}, 3 \mathrm{~A}$ e frutos totais, os genótipos não apresentaram diferenças estatísticas entre si, com exceção do Itaquiraí, que apresentou a menor produção nos quatros parâmetros avaliados (Tabela 3).

TABELA 1 - Números médios de frutos de primeira por planta ${ }^{1}$ (diâmetro equatorial $<65 \mathrm{~mm}$ ) para três níveis diferentes de adubação potássica em nove genótipos de maracujá azedo, Brasília, FAL-UnB, 2001.

\begin{tabular}{lccr}
\hline & \multicolumn{3}{c}{ Doses de $\mathrm{K}_{2} \mathrm{O}\left(\mathrm{kg} \mathrm{ha}^{-1}\right)$} \\
\hline \multicolumn{1}{c}{ GENÓTIPOS } & 0 & 640 & 1280 \\
\hline Híbrido EC-2-0 & $125,88 \mathrm{a}$ & $125,73 \mathrm{ab}$ & $118,69 \mathrm{a}$ \\
Marília Seleção Cerrado (MSC) & $92,44 \mathrm{ab}$ & $119,31 \mathrm{ab}$ & $120,46 \mathrm{a}$ \\
$\mathrm{F}_{1}$ (Roxo Fiji x Marília) & $118,26 \mathrm{ab}$ & $112,31 \mathrm{ab}$ & $122,04 \mathrm{a}$ \\
Porto Rico & $121,99 \mathrm{a}$ & $101,65 \mathrm{ab}$ & $94,30 \mathrm{a}$ \\
Vermelhão & $120,27 \mathrm{ab}$ & $132,27 \mathrm{a}$ & $105,07 \mathrm{a}$ \\
$\mathrm{F}_{1}$ (Marília x Roxo Australiano) & $92,83 \mathrm{ab}$ & $104,05 \mathrm{ab}$ & $87,40 \mathrm{a}$ \\
Redondão & $87,17 \mathrm{ab}$ & $84,42 \mathrm{~b}$ & $113,44 \mathrm{a}$ \\
IAC-273 & $88,41 \mathrm{ab}$ & $93,53 \mathrm{ab}$ & $105,97 \mathrm{a}$ \\
Itaquiraí & $79,69 \mathrm{~b}$ & $111,46 \mathrm{ab}$ & $95,20 \mathrm{a}$ \\
\hline F (genótipos x potássio) & $1,84^{*}$ & $1,84^{*}$ & $1,84^{*}$ \\
\hline DMS (5\%) & 24,03 & 24,03 & 24,03 \\
\hline Média & 102,99 & 109,41 & 106,95 \\
\hline Coef. de Variação (\%) & 17,32 & 17,32 & 17,32 \\
\hline
\end{tabular}

* Significativo a $5 \%$ de probabilidade pelo teste de Tukey.

Médias seguidas pela mesma letra nas colunas não diferem significativamente entre si a 5\% pelo teste de Tukey.

'Produção de onze colheitas semanais, a partir da décima semana após o início da colheita.

TABELA 2 -Número de frutos de primeira por planta (diâmetro equatorial $<65 \mathrm{~mm})$, frutos $1 \mathrm{~A}(65<\mathrm{DE}<75 \mathrm{~mm})$, frutos $2 \mathrm{~A}(75<\mathrm{DE}<85 \mathrm{~mm})$, frutos $3 \mathrm{~A}(\mathrm{DE}>85 \mathrm{~mm})$ e frutos totais (fruto com qualquer diâmetro) de nove genótipos de maracujá azedo, Brasília, FAL-UnB, 2001.

\begin{tabular}{|c|c|c|c|c|c|}
\hline GENÓTIPOS & $\mathrm{NFP}^{1}$ & NF1A & NF2A & $\mathrm{NF} \mathrm{A}^{\mathrm{I}}$ & $\mathrm{NFT}^{2}$ \\
\hline Híbrido EC-2-0 & $123,44 a$ & $21,53 a$ & $2,83 \mathrm{ab}$ & $3,23 \mathrm{ab}$ & $155,28 \mathrm{a}$ \\
\hline Marília Seleção Cerrado (MSC) & $110,73 \mathrm{abc}$ & $22,00 \mathrm{a}$ & $3,53 \mathrm{a}$ & $3,30 \mathrm{a}$ & $149,24 a$ \\
\hline $\mathrm{F}_{1}$ (Roxo Fiji x Marília) & $117,54 \mathrm{abc}$ & $19,96 \mathrm{a}$ & $2,77 \mathrm{ab}$ & $3,21 \mathrm{ab}$ & $139,84 \mathrm{ab}$ \\
\hline Porto Rico & $105,98 \mathrm{abc}$ & $19,94 \mathrm{a}$ & $3,07 \mathrm{a}$ & $3,24 a b$ & $136,70 \mathrm{ab}$ \\
\hline Vermelhão & $119,21 \mathrm{ab}$ & $15,93 \mathrm{ab}$ & $1,75 \mathrm{ab}$ & $3,20 \mathrm{ab}$ & $146,79 \mathrm{a}$ \\
\hline $\mathrm{F}_{1}$ (Marília x Roxo Australiano) & $94,76 \mathrm{c}$ & $20,63 a$ & $3,45 \mathrm{a}$ & $3,26 a b$ & $123,72 \mathrm{ab}$ \\
\hline Redondão & $95,01 \mathrm{c}$ & $15,82 \mathrm{ab}$ & $3,04 \mathrm{ab}$ & $3,27 \mathrm{ab}$ & $120,81 \mathrm{ab}$ \\
\hline IAC-273 & $95,97 \mathrm{bc}$ & $21,39 \mathrm{a}$ & $3,60 \mathrm{a}$ & $3,28 \mathrm{a}$ & $123,50 \mathrm{ab}$ \\
\hline Itaquiraí & $95,45 \mathrm{bc}$ & $10,56 b$ & $0,83 b$ & $3,17 b$ & $111,79 b$ \\
\hline F (genótipos) & $1,84^{*}$ & $5,21^{* *}$ & $3,40^{* *}$ & $3,16^{* *}$ & $3,76^{* *}$ \\
\hline DMS (5\%) & 24,03 & 7,50 & 2,24 & 0,10 & 34,62 \\
\hline Média & 106,46 & 18,65 & 2,76 & 3,24 & 134,19 \\
\hline Coef. De Variação (\%) & 17,32 & 30,88 & 62,17 & $2,45^{3}$ & 19,80 \\
\hline
\end{tabular}

*-**Significativo a $5 \%$ de probabilidade e a $1 \%$ de probabilidade pelo teste de Tukey, respectivamente.

Médias seguidas pela mesma letra nas colunas não diferem significativamente entre si a $5 \%$ pelo teste de Tukey.

'Produção de onze colheitas semanais, a partir da décima semana após o início da colheita.

${ }^{2}$ Produção de vinte colheitas semanais, a partir da primeira semana de colheita. ${ }^{3}$ Análise feita com dados transformados para raiz $(\mathrm{x}+10)$. 
TABELA 3 - Produção em $\mathrm{kg} \mathrm{ha}^{-1}$ de frutos de primeira (diâmetro equatorial $\left.<65 \mathrm{~mm}\right)$, frutos $1 \mathrm{~A}(65<\mathrm{DE}<75 \mathrm{~mm})$, frutos $2 \mathrm{~A}(75<\mathrm{DE}<85 \mathrm{~mm})$, frutos $3 \mathrm{~A}(\mathrm{DE}>85 \mathrm{~mm})$ e frutos totais (fruto com qualquer diâmetro) de nove genótipos de maracujá azedo, Brasília, FAL-UnB, 2001

\begin{tabular}{|c|c|c|c|c|c|}
\hline GENÓTIPOS & $\mathrm{FP}^{1}$ & $\mathrm{~F}_{1 \mathrm{~A}}{ }^{\mathrm{I}}$ & $\mathrm{F}^{2 \mathrm{~A}^{\mathrm{I}}}$ & $\mathrm{F}^{2} \mathrm{~A}^{\mathrm{T}}$ & $\mathrm{FT}^{2}$ \\
\hline Híbrido EC-2-0 & $15.376 \mathrm{a}$ & $4.081 \mathrm{a}$ & $770 \mathrm{ab}$ & $152 \mathrm{ab}$ & $21.675 \mathrm{a}$ \\
\hline Marília Seleção Cerrado (MSC) & $13.994 \mathrm{ab}$ & $4.587 \mathrm{a}$ & $1.006 \mathrm{a}$ & $269 \mathrm{a}$ & $21.577 \mathrm{a}$ \\
\hline $\mathrm{F}_{1}$ (Roxo Fiji x Marília) & $13.724 \mathrm{abc}$ & $3.658 \mathrm{ab}$ & $741 \mathrm{ab}$ & $140 \mathrm{ab}$ & $19.715 \mathrm{ab}$ \\
\hline Porto Rico & 13.184abc & $3.933 \mathrm{ab}$ & $848 \mathrm{ab}$ & $180 \mathrm{ab}$ & $19.518 \mathrm{ab}$ \\
\hline Vermelhão & $14.140 \mathrm{ab}$ & $3.241 \mathrm{ab}$ & $453 \mathrm{ab}$ & $81 \mathrm{ab}$ & $19.351 \mathrm{ab}$ \\
\hline $\mathrm{F}_{1}$ (Marília x Roxo Australiano) & $12.515 \mathrm{abc}$ & $4.190 \mathrm{a}$ & $959 \mathrm{a}$ & $211 \mathrm{ab}$ & $18.728 \mathrm{abc}$ \\
\hline Redondão & $11.713 \mathrm{bc}$ & $4.122 \mathrm{a}$ & $942 \mathrm{a}$ & $268 \mathrm{a}$ & $17.611 \mathrm{abc}$ \\
\hline IAC-273 & $10.890 \mathrm{c}$ & $3.690 \mathrm{ab}$ & $1.005 \mathrm{a}$ & $253 \mathrm{ab}$ & $16.582 \mathrm{bc}$ \\
\hline Itaquiraí & $10.897 \mathrm{c}$ & $2.408 \mathrm{~b}$ & $261 b$ & $24 b$ & $14.246 \mathrm{c}$ \\
\hline F (genótipo) & $5,53^{* *}$ & $3,16^{* *}$ & $3,18^{* *}$ & $2,79^{* *}$ & $4,85^{* *}$ \\
\hline DMS (5\%) & 2.974 & 1.617 & 668 & 36 & 4.840 \\
\hline Média & 12.937 & 3.768 & 776 & 175 & 18.778 \\
\hline Coef. de Variação (\%) & 17,64 & 32,92 & 65,09 & $0,87^{3}$ & 19,78 \\
\hline
\end{tabular}

**Significativo a $1 \%$ de probabilidade pelo teste de Tukey.

Médias seguidas pela mesma letra nas colunas não diferem significativamente entre si a 5\% pelo teste de Tukey.

${ }^{1}$ Produção de onze colheitas semanais, a partir da décima semana após o início da colheita.

${ }^{2}$ Produção de vinte colheitas semanais, a partir do inicio da colheita.

${ }^{3}$ Transformado para raiz $(\mathrm{x}+10)$.

Para a variável peso médio dos frutos, $\mathrm{o}_{1}$ (Marília $\mathrm{x}$ Roxo Australiano) foi o que apresentou o maior peso médio para frutos de primeira e frutos totais, não diferindo, porém, dos genótipos MSC, híbrido EC-2-0, Porto Rico e Redondão para peso médio de frutos totais e, além destes, do genótipo Vermelhão para peso médio de frutos de primeira. $\mathrm{Na}$ avaliação do peso médio de frutos $1 \mathrm{~A}, 2 \mathrm{~A}$ e $3 \mathrm{~A}$, os genótipos não apresentaram diferenças estatísticas entre si. (Tabela 4).

Considerando-se os cinco meses de colheita, os resultados obtidos estão acima da média nacional e foram proporcionalmente iguais e superiores a vários resultados citados na literatura. As diferenças ocorridas são devidas, principalmente, aos diferentes materiais genéticos e às diferentes condições climáticas e edáficas. Para o peso médio de frutos, muito embora, a adubação potássica não tenha influenciado os resultados, os genótipos apresentaram diferenças significantes entre si, variando até 15,42 g do maior [ $\mathrm{F}_{1}$ (Marília x Roxo Australiano - 103,42 g)] ao menor (Itaquiraí - $88 \mathrm{~g}$ ). Faria et al. (1987), ao avaliarem a resposta do maracujazeiro amarelo à adubação com N, $\mathrm{P}$ e K, obtiveram a produção média de 20,8 $\mathrm{t} \mathrm{ha}^{-1}$, 9,2 $\mathrm{t} \mathrm{ha} \mathrm{h}^{-1} \mathrm{e} 14,6 \mathrm{tha} \mathrm{ha}^{-1} \mathrm{e}$ os pesos médios de frutos de $100,1 \mathrm{~g}, 72,0 \mathrm{~g}$ e 100,6 g, para os segundo, terceiro e quarto ano de produção, respectivamente. Borges et al. (1998), em cinco meses de produção do maracujá amarelo, verificaram que as doses de N, P e K não influenciaram a produtividade, que teve uma média de $7,4 \mathrm{t} \mathrm{ha}^{-1}$. Oliveira et al. (1998) obtiveram com presença e ausência de adubação, em condições de sequeiro, pesos médios de frutos variando de 72 a $88 \mathrm{~g}$. Sanzonowicz et al. (2000) não verificaram diferenças estatísticas significativas ao utilizarem cinco doses diferentes de adubação potássica na produção total de frutos do maracujazeiro amarelo. As equações de regressão para a produção de frutos de primeira por hectare, produção total de frutos ha-1 ${ }^{-1}$, peso médio de frutos de primeira e peso médio de frutos $1 \mathrm{~A}$ de frutos em função das doses de potássio aplicadas possuem representação do tipo linear (Figuras 1, 2, 3 e 4). Conforme as equações com aumento de $640 \mathrm{~kg}$ de $\mathrm{K}_{2} \mathrm{O}$ ha $^{-1}$, esperava-se aumento médio de $632,25 \mathrm{~kg}$ frutos de primeira ha-1, $1.007 \mathrm{~kg}^{-10}$ total de frutos ha-1 $, 2,35 \mathrm{~g} \mathrm{de}$ peso médio/fruto de primeira e $4,20 \mathrm{~g}$ de peso médio/fruto $1 \mathrm{~A}$. As correlações que mostraram-se positivamente fortes foram: Produção de frutos de primeira $\left(\mathrm{kg} \mathrm{ha}^{-1}\right)$ com produção total de frutos $\left(\mathrm{kg} \mathrm{ha}^{-1}\right)$, número de frutos de primeira e número de frutos totais; produção de frutos $1 \mathrm{~A}$ com produção de frutos $2 \mathrm{~A}$, produção de frutos $3 \mathrm{~A}$, produção total de frutos, número de frutos $1 \mathrm{~A}$ e número de frutos $2 \mathrm{~A}$; produção de frutos $2 \mathrm{~A}$ com produção de frutos $3 \mathrm{~A}$, número de frutos $2 \mathrm{~A}$ e número de frutos $3 \mathrm{~A}$; produção de frutos $3 \mathrm{~A}$ com número de frutos $2 \mathrm{~A}$ e número de frutos $3 \mathrm{~A}$; produção total de frutos com número de frutos de primeira e número total de frutos; número de frutos de primeira com número de frutos totais; número de frutos $2 \mathrm{~A}$ com número de frutos $3 \mathrm{~A}$ e peso médio de frutos de primeira com peso médio total dos frutos.

TABELA 4 - Peso médio em gramas de frutos de primeira (diâmetro equatorial $<65 \mathrm{~mm})$, frutos $1 \mathrm{~A}(65<\mathrm{DE}<75 \mathrm{~mm})$, frutos $2 \mathrm{~A}(75<\mathrm{DE}<85 \mathrm{~mm})$, frutos $3 \mathrm{~A}(\mathrm{DE}>85 \mathrm{~mm})$ e frutos totais (fruto com qualquer diâmetro) de nove genótipos de maracujá azedo, Brasília, FAL-UnB, 2001

\begin{tabular}{|c|c|c|c|c|c|}
\hline GENÓTIPOS & PMFP $^{1}$ & $\mathrm{PMF} \mathrm{A}^{1}$ & PMF2A $^{1}$ & PMF3A $^{1}$ & $\mathrm{PMFT}^{2}$ \\
\hline Híbrido EC-2-0 & $86,04 a b c$ & $144,87 \mathrm{a}$ & $188,68 \mathrm{a}$ & $227,91 \mathrm{a}$ & $96,34 \mathrm{abc}$ \\
\hline Marília Seleção Cerrado (MSC) & $86,48 \mathrm{ab}$ & $141,69 \mathrm{a}$ & $197,45 \mathrm{a}$ & $259,77 \mathrm{a}$ & $100,04 a b$ \\
\hline $\mathrm{F}_{1}$ (Roxo Fiji x Marília) & $80,23 \mathrm{bc}$ & $141,26 \mathrm{a}$ & $186,57 \mathrm{a}$ & $260,64 a$ & $91,81 b c$ \\
\hline Porto Rico & $85,73 a b c$ & $147,21 \mathrm{a}$ & $188,29 a$ & $246,39 a$ & $98,26 a b c$ \\
\hline Vermelhão & $82,44 a b c$ & $142,75 a$ & $179,44 a$ & $262,67 \mathrm{a}$ & $91,42 \mathrm{bc}$ \\
\hline $\mathrm{F}_{1}$ (Marília x Roxo Australiano) & $89,96 \mathrm{a}$ & $150,68 \mathrm{a}$ & $194,54 \mathrm{a}$ & $256,44 \mathrm{a}$ & $103,42 \mathrm{a}$ \\
\hline Redondão & $84,81 \mathrm{abc}$ & $151,70 \mathrm{a}$ & $206,23 a$ & $254,83 \mathrm{a}$ & $99,34 \mathrm{abc}$ \\
\hline IAC-273 & $78,01 \mathrm{c}$ & $142,78 \mathrm{a}$ & $187,14 \mathrm{a}$ & $235,25 a$ & $91,39 b c$ \\
\hline Itaquiraí & $78,66 \mathrm{bc}$ & $153,18 \mathrm{a}$ & $206,36 a$ & $249,72 \mathrm{a}$ & $88,00 \mathrm{c}$ \\
\hline F (genótipo) & $4,65^{* *}$ & $1,40 \mathrm{~ns}$ & $2,28 \mathrm{~ns}$ & $1,72 \mathrm{~ns}$ & $4,02^{* *}$ \\
\hline DMS (5\%) & 8,40 & 17,58 & 27,43 & 41,25 & 11,52 \\
\hline Média & 83,60 & 146,24 & 192,75 & 250,41 & 95,56 \\
\hline Coef. de Variação (\%) & 7,71 & 9,22 & 10,92 & 12,64 & 9,24 \\
\hline
\end{tabular}

**Significativo a $1 \%$ de probabilidade pelo teste de Tukey. ns - não significativo.

Médias seguidas pela mesma letra nas colunas não diferem significativamente entre si a 5\% pelo teste de Tukey.

'Produção de onze colheitas semanais, a partir da décima semana após o início da colheita.

${ }^{2}$ Produção de vinte colheitas semanais, a partir do início da colheita. 


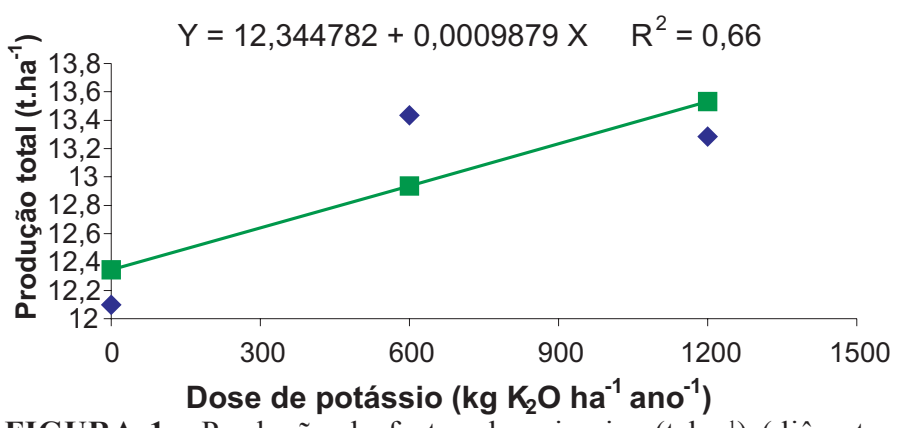

FIGURA 1 - Produção de frutos de primeira $\left(\mathrm{t} \mathrm{ha}^{-1}\right)$ (diâmetro equatorial $<65 \mathrm{~mm}$ ) de maracujá azedo em função da aplicação de doses crescentes de potássio, Brasília, FAL/ $\mathrm{UnB}, 2001$.

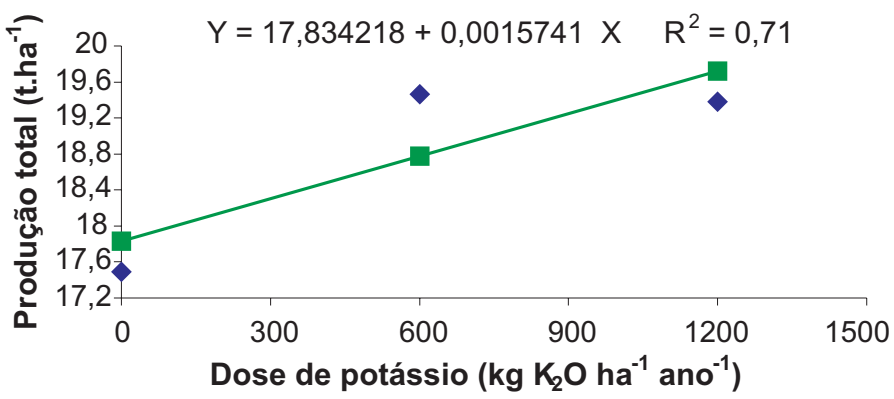

FIGURA 2 - Produção total $\left(\mathrm{t} \mathrm{ha}{ }^{-1}\right)$ de frutos de maracujá azedo em função da aplicação de doses crescentes de potássio, FAL/UnB, 2001.

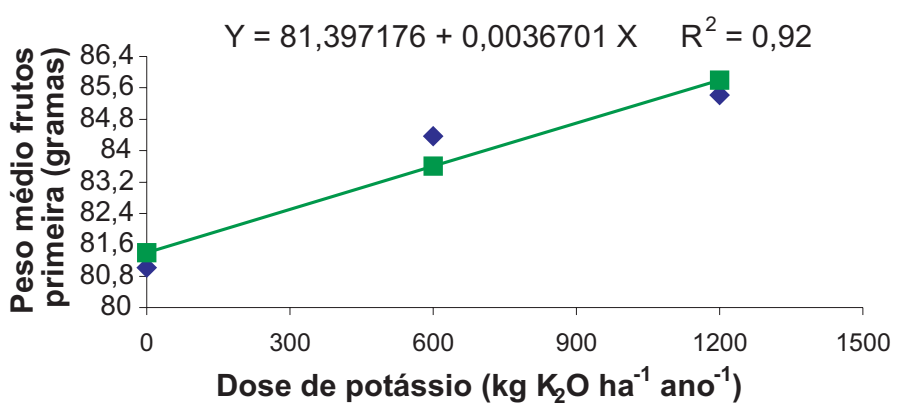

FIGURA 3 - Peso médio (g) de frutos de primeira (diâmetro equatorial $<65 \mathrm{~mm}$ ) de maracujá azedo em função da aplicação de crescentes doses de potássio. Brasília, FAL/UnB, 2001.

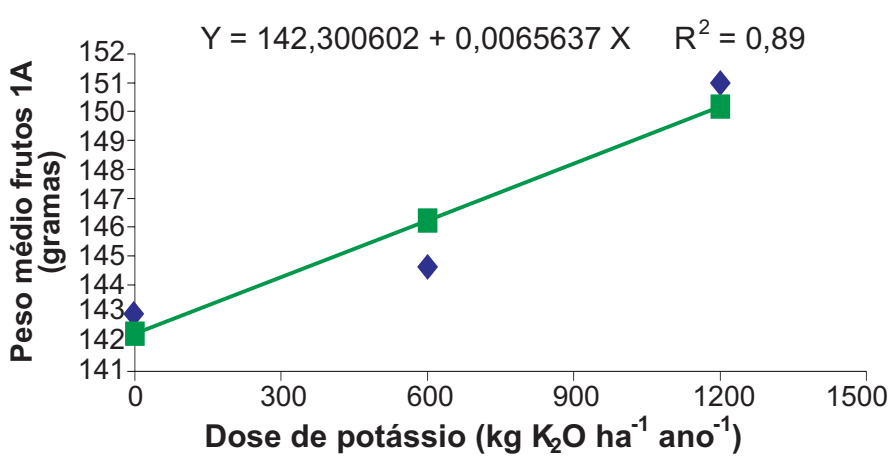

FIGURA 4 - Peso médio $(\mathrm{g})$ de frutos $1 \mathrm{~A}(65<$ diâmetro equatorial $<75 \mathrm{~mm})$ de maracujá azedo em função da aplicação de crescentes doses de potássio. Brasília, FAL/UnB, 2001.

\section{CONCLUSÕES}

1) Os genótipos híbrido EC-2-0 e Marília Seleção Cerrado (MSC) foram os que apresentaram as maiores produtividades, superando $20 \mathrm{t} /$ ha.

2) A adubação potássica influenciou a produção total de frutos e de primeira ha ${ }^{-1}$, a produção de frutos totais ha $^{-1}$, o peso médio de frutos de primeira e o peso médio de frutos $1 \mathrm{~A}$.

\section{REFERÊNCIASBIBLIOGRÁFICAS}

BORGES, A. L.; LIMA, A. de A.; CALDAS, R. C. Nitrogênio, fósforo e potássio na produção e qualidade dos frutos de maracujá-amarelo no primeiro ano. In: SIMPÓSIO BRASILEIRO SOBREA CULTURA DO MARACUJAZEIRO, 5., 1988, Jaboticabal. Anais... Jaboticabal: FUNEP, 1998. p.340-342.

FARIA, J.L. C.; MANICA, I.; COLAUTO, N.M.; STRONSKI, M. do S.; APEEL, H.B. Resposta do maracujazeiro amarelo (Passiflora edulis Sims f. flavicarpa Deg.) à adubação com N, P e K, no segundo, terceiro e quarto anos de produção. Revista Brasileira de Fruticultura, Cruz das Almas, v. 9, n. 3, p. 45-50, 1987.

GUERRA, N.B.; LIVERA, A.V.S. Correlação entre o perfil sensorial e determinações físicas e químicas do abacaxi cv. Pérola. Revista Brasileira de Fruticultura, Cruz das Almas, v. 21, n. 1, p.32-35, 1999.

HAAG, H.P.; OLIVEIRA, G.D.; BORDUCHI, A.S.; SARRUGE, J.R. Absorção de nutrientes por duas variedades de maracujá. In: CONGRESSO BRASILEIRO DE FRUTICULTURA, 1973, Viçosa. Anais... Viçosa: Sociedade Brasileira de Fruticultura, 1973. v.30, p.267279.

JUNQUEIRA, N.T.V.; ICUMA, I.M.;VERAS, M.C.M.; OLIVEIRA, M.A.S.; ANJOS, J.R.N. dos. Cultura do maracujazeiro. In: SILVA, J.M. de M. (Ed.) Incentivo à fruticultura no Distrito Federal: Manual de fruticultura. 2. ed. Brasília: OCDF, COOLABORA, 1999. p. 42-52.

MANICA, I. Fruticultura tropical 1. Maracujá. São Paulo: Ed. Agronômica Ceres, $1981.151 \mathrm{p}$.

MELETTI, M.L.M. Maracujá: Produção e comercialização. Campinas: IAC, 1999. 64p. (Boletim Técnico, 181).

OLIVEIRA, F.K.D.; DANTAS, J.P.; SILVA, A.Q. da; LIMA, E.M. de; CAVALCANTE, L.F. Produtividade e estado nutricional em plantas de maracujá amarelo. In: SIMPÓSIO BRASILEIRO SOBRE A CULTURA DO MARACUJAZEIRO, 5., 1998, Jaboticabal. Anais... Jaboticabal: Funep, 1998. p.337-339.

OSAKI, F. Calagem e adubação. 2. ed. Campinas: Instituto Brasileiro de Ensino Agrícola, 1991. 503p.

QUAGGIO, J.A.; PIZA JUNIOR. C.T. Nutrição mineral e adubação da cultura do maracujá. In: SIMPÓSIO BRASILEIRO SOBRE A CULTURA DO MARACUJAZEIRO, 5., 1998, Jaboticabal. Anais... Jaboticabal: Funep, 1998. p.130-156.

RAIJ, B. Van.; CANTARELLA, H.; QUAGGIO, J.A.; FURLANI, A.M.C. Recomendações de adubação e calagem para o Estado de São Paulo. 2., ed. Campinas: Instituto Agronômico \& Fundação IAC, 1996. 285p. (Boletim Técnico 100).

RUGGIERO, C.; JOSÉ,A.R.S.;VOLPE, C.A.; OLIVEIRA, J.C.; DURIGAN, J.F.; BAUMGARTNER, J.G; SILVA, J.R. da.; NAKAMURA, K.; FERREIRA, M.E. Maracujá para exportação: aspectos técnicos da produção.Brasília: MAARA;SDR;EMBRAPA-SPI, 1996.40p.

SANZONOWICZ, C.; SILVA, R.P. da; PEIXOTO, J.R.; JUNQUEIRA, N.T.V.; OLIVEIRA, J.A. de. Efeito da adubação potássica na produção do maracujazeiro amarelo (Passiflora edulis Sims f. flavicarpa Deneger). In: CONGRESSO BRASILEIRODE FRUTICULTURA, 16. Fortaleza. Resumos... Fortaleza: Sociedade Brasileira de Fruticultura, 2000. p. 454.

SOUZA, M. de; A.C.; GUIMARÃES, P.T.G.; CARVALHO, J.G. de; FRAGOAS, J.C. Maracujazeiro. In: COMISSÃO DE FERTILIDADE DOSOLO DOESTADODE MINAS GERAIS. Recomendações para o uso de corretivos e fertilizantes em Minas Gerais $-5^{\text {a }}$ aproximação. Viçosa, 1999. 359p.

ZONTA, E.P.; MACHADO, A.A. Sistema de análises estatísticas (SANEST) para microcomputadores. In: SIMPÓSIO DE ESTATÍSTICAAPLICADA À EXPERIMENTAÇÃO, 1995, Piracicaba. Resumos... Campinas: Fundação Cargill, 1995. P17-18. 